\title{
Review of real brain-controlled wheelchairs
}

\author{
Á. Fernández-Rodríguez, F. Velasco-Álvarez and R. Ron-Angevin \\ Department of Electronic Technology, University of Malaga, 29071, Málaga, Spain.
}

afernandezrguez@uma.es; fvelasco@uma.es; rron@uma.es

\begin{abstract}
This paper presents a review of the state of the art regarding wheelchairs driven by a brain-computer interface (BCI). Using a brain-controlled wheelchair (BCW), disabled users could handle a wheelchair through their brain activity, granting autonomy to move through an experimental environment. A classification is established, based on the characteristics of the $\mathrm{BCW}$, such as the type of electroencephalographic (EEG) signal used, the navigation system employed by the wheelchair, the task for the participants, or the metrics used to evaluate the performance. Furthermore, these factors are compared according to the type of signal used, in order to clarify the differences among them. Finally, the trend of current research in this field is discussed, as well as the challenges that should be solved in the future.
\end{abstract}

Keywords: brain-computer interface, wheelchair, real environment, review.

\section{Introduction}

One of the main objectives of research groups working on assistive technologies is to improve the life quality and autonomy of people affected by motor neuron diseases (MND), such as amyotrophic lateral sclerosis (ALS). A brain-computer interface (BCI) is a tool to establish an additional communication channel between the user and a particular device, through their brain activity (1). Therefore, numerous applications are proposed for these users: e.g., managing a speller matrix (2), a robotic arm (3), a telepresence robot (4), or a domotic system (5), as well as applications focused on neurorehabilitation (6). Through these interfaces, people affected by a MND could gain some autonomy by means of the addition of this new communication channel that does not require the use of the motor system. Several types of BCI exist, depending on various features that will be briefly explained below. One of the most important features is the recording technique of the physiological signal, such as electroencephalography (EEG), functional magnetic resonance imaging (fMRI) or nearinfrared spectroscopy (fNIRS). However, the most used physiological signal is the EEG, mainly because of its adequate temporal resolution, portability, and relative low cost (7). There are also numerous types of EEG signal that can be recorded. Initially, we can distinguish between an endogenous signal, which is evoked at will by the participant, or an exogenous signal, which is evoked by an external stimulus presentation (8). Among the endogenous signals, the slow cortical potentials (SCP) are produced by a change in the level of cortical activity (9), Event-Related Desynchronization/Synchronization (ERD/ERS) changes elicited by motor imagery (MI) tasks, and other signals corresponding to different mental tasks, such as objects' mental rotation or word association (10). On the other hand, regarding exogenous signals, the P300 signal is a positive peak that appears in the EEG approximately $300 \mathrm{~ms}$ after the presentation of a rare stimulus (2); the steady-state visual evoked potentials (SSVEP) are changes in the neural activity located at the visual cortex that occur at the same frequency as a blinking stimulus (11).
Regarding the kind of electrode, there are two types: dry and wet. The second needs an electrolytic gel to record the signal. Most electrodes used in laboratories are wet due to a higher quality reception of the EEG signal because of lower impedance (12). These electrodes are placed at the scalp following the International 10/20 system which specific configuration and number of electrodes depends of technique factors, as the EEG signal to register, or practical factors, as the time required for the set up. Nevertheless, in recent years, many brands have marketed dry electrodes (e.g. Neurosky, Emotiv Systems or OCZ Technology) that have come to be used in some studies to prove their accuracy, and to see whether they might be suitable for safe operation for people with MND (e.g. $(13,14))$.

The use of BCI systems for navigation of multiple devices - in both real and virtual environments - has been the subject of numerous investigations. Navigation in virtual environments can be for simple tasks such as moving a car to the right or left of the road (15), or more complex ones, such as the management of a character in a videogame (16). The use of these interfaces in real environments, which are less controlled and dependent on the use of devices that interact with them, may be more challenging, because mistakes can have real consequences for users. However, the development of these applications can be more useful, because they allow direct user intervention in their environment through their brain signal. Furthermore, as specified by Millán et al. (17): "BCIs must be combined with existing assistive technologies (AT), especially those they already utilize", so a braincontrolled wheelchair (BCW) would be a perfect example of this combination.

The first BCW was published by Tanaka et al. in 2005 (18). One could cite as a precursor the paper that of Millán et al. (19), in which the ability to manage a small mobile robot using an EEG signal was shown, setting a precedent for the control of a larger real system, such as a robotic wheelchair. Nevertheless, the leap from a remotely managed device to a wheelchair needs to be done carefully, as an accurate system is needed in which the safety of the user is not in danger. Therefore, the proposals to control a robotic wheelchair must 
overcome some challenges to improve the quality of life of patients with severely impaired motor abilities. First, one of the issues to be faced is the use of reliable navigation systems to ensure the user safety and offer a flexible displacement in the environment so that the user can move comfortably and freely. In this way, it could be interesting to show the most useful and innovative proposals that could be used in future prototypes. Similarly, it should not be forgotten that the target population of these interfaces are patients with severely impaired motor abilities, so that the proposals should be adapted to these users and offer them an experience as pleasant as possible. Finally, due to the numerous proposals made in this area, it would be desirable to detail how the performance was evaluated and what were the participants' characteristics (e.g. the number of users who tried to control the wheelchair and complete the tasks, if they were trained or had some kind of disease).

In 2013, Bi et al. (20) published a survey of BCIcontrolled mobile robots; however, the present paper will focus on the particular field of BCWs, including the development and characteristics of the different proposals tested in real environments to date (figure 1), describing the type of signal that was used to control the navigation system device, users who handled it, the tasks performed by them, the navigation of the interface, and the metrics used to evaluate the performance. Therefore, this review includes papers that use a BCI system to control a wheelchair in a real environment, and that detail the $\mathrm{BCW}$ with enough data to be classified, based on the mentioned characteristics. The different interfaces are compared according to the signal used, in order to highlight the advantages and disadvantages between them. Finally, it is important to advise that in the case to find similar BCWs and authorship, we only include the most detailed paper.

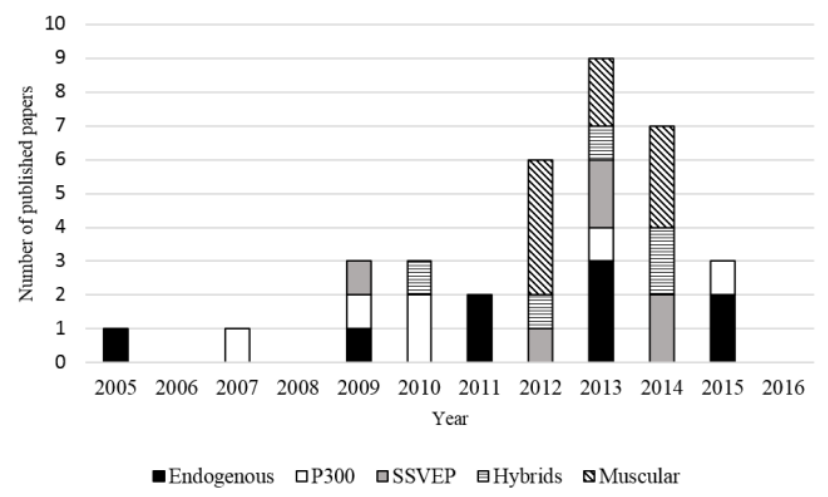

Figure 1. BCW papers compiled in this review.

\subsection{Glossary}

In this brief section, we will define some terms used in the rest of the paper. Even when most of them are commonly used terms, it is worth defining them clearly in order to avoid any ambiguity.

- User's tasks: In order to control a BCI, users perform different tasks whose consequences are predictable, so that they can be used as inputs. These control tasks include mental strategies (such as the MI of limbs or selective attention tasks) (21), but actual muscular tasks as well, being that some BCI systems are assisted by real movements.

- P300 BCW and SSVEP BCW: wheelchairs that only rely on P300 and SSVEP, respectively.

- ERD/ERS BCW: wheelchairs that depend on the users' control of their electrophysiological activity through the execution of mental tasks that affect the EEG causing ERD/ERS changes. The used mental tasks include tasks such as MI, mental calculations, or word association (10). It should be noted that a BCW that analyzes the EEG caused by actual movements (not MI) will be classified in this paper as "muscle-assisted" (see next categories) for comparison purposes.

- Hybrid BCIs are commonly accepted as systems relying on one EEG input combined with one or more channels (that can be EEG, electromyography (EMG), electrooculography (EOG) or movement detection, among others). However, as one of the focuses of the paper is to compare parameters of similar systems, we have defined in this paper a subgroup of hybrids systems so that motor actions were excluded (see next definition).

- Hybrid-mental BCW: wheelchairs that are based on more than one kind of EEG signal (e.g. ERD/ERS and P300), as a consequence of different mental strategies, excluding any kind of real motor action.

- Muscle-assisted BCW. As mentioned above, we think that systems using motor actions (even when analyzing their consequent EEG) should not be compared with those that use purely mental tasks. For this reason, we have included in this group called Muscle-assisted BCW two kinds of wheelchairs: i) those that use EEG signals elicited by actual motor execution; and ii) hybrid wheelchairs that, in addition to purely cognitive tasks, use muscular activity as information input (detected by means of EEG artifact, EMG, or EOG).

- Low-level navigation: the control of the wheelchair is achieved through simple navigation commands, such as "move forward" or "turn right", and basic supports as stopping the wheelchair when obstacles are encountered. In this way, users can perform any path they want to, having fine control of the specific movement. The system does not assist the execution of the selected command.

- High-level navigation: these systems let users have a rough control of the $\mathrm{BCW}$, selecting high-level commands such as "take me to the kitchen" or "leave this room." The BCW must be equipped with some intelligence so that the specific path to the selected objective is transparent to users (in other words, the user does not select specific low-level commands).

- Shared-control navigation: both the user and the system share the control of the BCW $(22,23)$. This can be done in two ways: i) users generate low-level commands, while the system assists the navigation with features such as obstacle avoidance, or maximum likelihood command execution; and ii) users can switch between a low- and a high-level navigation mode.

- Discrete control: the selection of a navigation command implies a prefixed movement, e.g. a turn of 45 or 90 degrees or a fixed advance distance of $1 \mathrm{~m}$.

- Continuous control: the user can control the extension of the movement after the selection of a navigation command, e.g. the turn amplitude or the advance length. 
Usually, the movement continues as long as the user keeps the command active.

\section{State of the art in $\mathrm{BCW}$}

The use of invasive methods for capturing signals in BCI systems is less extended than non-invasive methods, i.e. the EEG (7). In the case of BCW, all the references cited in this review used EEG (figure 2). The different $\mathrm{BCW}$ papers have been divided regarding the signal used as input, so that five categories can be established: ERD/ERS, P300, SSVEP, hybrid-mental and muscle-assisted $\mathrm{BCW}$. The number of BCWs found for each category is: 9, 6, 6, 5 and 9, respectively. It is worth mentioning the absence of SCP-based systems, possibly due to the low information transfer rate (ITR), and the need for a longer training time to acquire control, even compared with ERD/ERS (24).

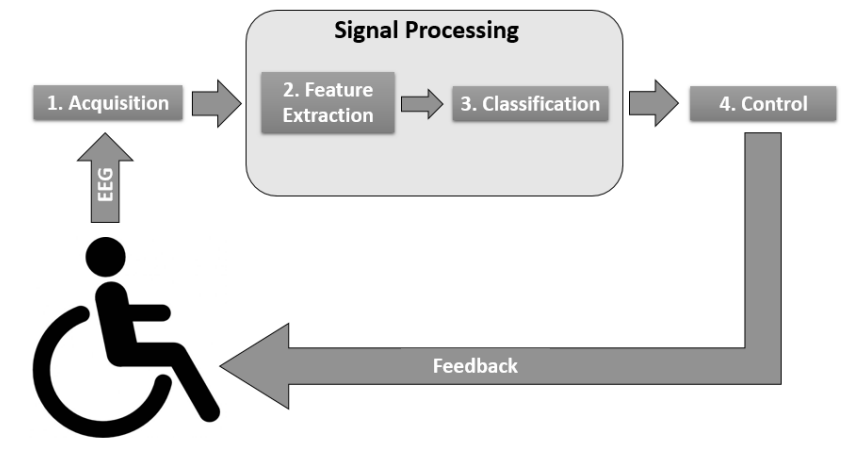

Figure 2. General model of a BCW. 1) The EEG is captured through electrodes at the Acquisition stage. 2) The raw signal is analyzed to obtain some significant characteristics called Features. 3) The main part of the Signal Processing uses the previous features to establish a Classification of the signals into a minimum of two groups; at this point the BCI system decides what state corresponds to the current EEG. 4) After classifying the signals, the system actuates in the Control stage moving the wheelchair. This movement is a Feedback for the subjects that helps them on the control of their EEG signals and, consequently, on the control of the BCW.

Within each type of EEG signal, the navigation system used by the BCW (which may be low-level management, high-level or shared control) is detailed. Then we will describe the most important features related to the participants (e.g. users affected by MND) (table 1). Next, the characteristics of the interface will be explained, referring to the commands available to the user (e.g. turn left/right, go forward or stop) and the tasks that must be performed for the selection of a command; i.e., voluntary mental tasks, selective attention to the stimulus, or muscle action (table 2).

Regarding the number of user tasks, these should be as few as possible, because the handling by the user will be simpler and, in the case of tasks that modulate ERD/ERS signals, fewer mistakes will be made in the classification $(25,26)$. On the other hand, it is of interest to maximize the number of commands, as they are the options available to the user to handle the BCW and move around the environment autonomously. Therefore, using a ratio between the number of available commands (AC) and the number of user tasks (UT) it is proposed, the command to task ratio (CTR):

$$
C T R=\frac{A C}{U T}
$$

It is worth mentioning that this ratio is not suitable for comparing BCI systems based on different EEG signals, as the tasks to be performed are be different cognitive processes. However, in the case of systems with similar EEG input, this ratio points out how easily new commands can be added.

To continue, the extraction features and classification methods used to categorize the different user's task in each signal category will be mentioned (table 3 ). Finally, the metrics used for the evaluation of user performance in handling the $\mathrm{BCW}$, such as the accuracy or the usability, are mentioned (table 4).

\subsection{ERD/ERS BCWS}

ERD/ERS signals are those elicited at the user's will by certain tasks that cause a variation in the amplitude of the neuronal rhythmic activity (8). However, learning to modulate these signals on a voluntary basis is usually complex, requiring more learning time for users than other systems (7). Despite this difficulty, as Nicolas-Alonso and Gomez-Gil (7) showed, the use of these signals has several advantages that should not be overlooked, such as: i) they are independent of any stimulation; ii) they can be operated by free will; iii) they are useful for users with affected sensory organs; and iv) they are suitable for cursor control.

\subsubsection{Navigation. Five BCWs based on ERD/ERS} signal had a low-level navigation system $(18,34,43,44,57)$, three used a shared management $(28,35,46)$ and one with a highlevel navigation system (59). Therefore, it can be seen that the handling of low-level is used to a greater degree than shared control or highlevel. The ERD/ERS signals may be of particular interest in low and shared navigation because they can offer continuous control of the BCW with a few low-level commands (e.g. forward, back or stop, turn left and turn right), to allow the free movement of the chair through the environment (60). However, thanks to the shared-control systems presented here, the management of the chair is assisted by various tools, such as obstacle detection and dodge, or assistance in selecting the most appropriate command, depending on the specific situation in the environment.

2.1.2 Participants. Referring to publications with ERD/ERS signal BCWs, these studies had an average of 3.22 participants $(\sigma=1.39)$. It is noteworthy that no study presented participants affected by MND. This low number of participants may be related to the difficulty in acquiring a proper and safe operation of a BCW which could imply an extensive training based on the ERD/ERS modulation of the EEG signal, as well as difficulties aggravated in the case of users with MND (e.g. security, communication, mobility and placement of instrumentation). Furthermore, the control of ERD/ERS signals may require a long training for some users. 
Table 1. Compiled papers and main characteristics in chronological order.

\begin{tabular}{|c|c|c|c|c|c|}
\hline $\mathrm{BCW}$ & Year & Signal & Navigation system & Control & Subjects \\
\hline$(18)$ & 2005 & ERD/ERS & Low-level & Discrete & 6 \\
\hline (27) & 2007 & P300 & High-level & Discrete & 5 \\
\hline (28) & 2009 & ERD/ERS & Shared & Continuous & 3 \\
\hline (29) & 2009 & P300 & Shared & Discrete & 5 \\
\hline (30) & 2009 & SSVEP & Shared & Discrete & 9 \\
\hline (31) & 2010 & ERD/ERS and P300 & High-level & Discrete & 5 \\
\hline (32) & 2010 & P300 & High-level & Discrete & $1^{\mathrm{a}}$ \\
\hline (33) & 2010 & P300 & Low-level & Discrete & 1 \\
\hline (34) & 2011 & ERD/ERS & Shared & Mixed $^{b}$ & 4 \\
\hline (35) & 2011 & ERD/ERS & Shared & Discrete & 2 \\
\hline (36) & 2012 & ERD/ERS and EMG & High-level & Continuous & 3 \\
\hline (37) & 2012 & ERD/ERS & Low-level & Not specified & 1 \\
\hline (38) & 2012 & Alpha band and EEG artifact & Low-level & Mixed $^{\mathrm{b}}$ & 7 \\
\hline (39) & 2012 & ERD/ERS and P300 & Low-level & Continuous & 2 \\
\hline$(40)$ & 2012 & P300 and EEG artifact & The user can choose low or high-level & Discrete & 4 \\
\hline (41) & 2012 & SSVEP & Low-level & Continuous & 2 \\
\hline$(42)$ & 2013 & EEG artifact & High-level & Discrete & 1 \\
\hline$(43)$ & 2013 & ERD/ERS & Low-level & Discrete & 1 \\
\hline (44) & 2013 & ERD/ERS & Low-level & Continuous & 3 \\
\hline$(45)$ & 2013 & ERD/ERS and EMG & Low-level & Continuous & 1 \\
\hline$(46)$ & 2013 & ERD/ERS & Shared & Continuous & 4 \\
\hline (47) & 2013 & P300 & Shared & Discrete & $11^{\mathrm{c}}$ \\
\hline$(48)$ & 2013 & SSVEP & Low-level & Discrete & 1 \\
\hline (49) & 2013 & P300 and SSVEP & Low-level & Continuous & 5 \\
\hline (50) & 2013 & SSVEP & Low-level & Continuous & $13^{\mathrm{d}}$ \\
\hline$(51)$ & 2014 & Alpha band & Low-level & Discrete & 8 \\
\hline$(52)$ & 2014 & ERD/ERS, EOG and P300 & Low-level & Continuous & 4 \\
\hline (53) & 2014 & ERD/ERS and SSVEP & Low-level & Mixed $^{\mathrm{b}}$ & 3 \\
\hline (54) & 2014 & ERD/ERS and SSVEP & Low-level & Continuous & 3 \\
\hline$(55)$ & 2014 & SSVEP & Shared & Continuous & 4 \\
\hline (56) & 2014 & EOG (embedded in EEG) & Low-level & Continuous & 5 \\
\hline$(57)$ & 2015 & ERD/ERS & Low-level & Discrete & 3 \\
\hline$(58)$ & 2015 & SSVEP & High-level & Discrete & 37 \\
\hline (59)a & 2016 & ERD/ERS & High-level & Discrete & 3 \\
\hline$(59) b$ & 2016 & P300 & High-level & Discrete & 6 \\
\hline
\end{tabular}

${ }^{a}$ affected by Guillain-Barre Syndrome

${ }^{\mathrm{b}}$ discrete turns and continuous advance and recoil

c 1 participant with cerebral palsy and motor impairment

d 1 paraplegic participant

2.1.3 Task and interface. The average of tasks and commands used was $2.67(\sigma=0.71)$ and $5.77(\sigma$ $=8.06)$, respectively. So the CTR was $2.11(\sigma=$ 2.67). It must be taken into account that the BCW of the study of Varona-Moya et al. (57) Zhang et al. (59) was the only ones with a CTR greater than the unit. The rest of the studies have a CTR equal to the unit, where every task served to execute a single command. The specific tasks to be performed by the user were fairly homogeneous across studies. Most of the papers $(80 \%)$ used hand MI - left, right, or both - as one of its tasks. It was also common to use feet MI or to maintain a state of rest. On the other hand, the most common commands were to move forward, and to rotate the chair to the left and right. Just one of the BCWs used a graphical user interface (GUI), the proposal of Zhang et al. (59) that was the one with the largest number of commands through the use of a successive dichotomy method. By other side, other article includes the presence of an audio interface, indicating different commands serially that users could select through righthand MI (57). Hence, the only two proposal based purely on ERD/ERS signal whose CTR outperformed the unit were those that needed a specific graphical or auditory serial interface.

2.1.4 Feature extraction and classification methods. The feature extraction methods used were quite heterogeneous; however, the power spectral density (PSD) can be highlighted as being the most used by the proposals with ERD/ERS 
Table 2. User's tasks, used commands, and command tot task ratio for the brain-controlled wheelchairs compiled in this review.

\begin{tabular}{|c|c|c|c|c|}
\hline & Paper & User's task & Commands & CTR \\
\hline \multirow{9}{*}{ ERD/ERS } & (18) & $2 ;$ left/right thinking & 2 ; forward in diagonal line left/right & 1 \\
\hline & $(28)$ & 3; MI left hand, word association, relax or arithmetic operation & 3 ; forward and turn left/right & 1 \\
\hline & (34) & 4; MI left/right hand and foot, and idle state & 4 ; forward, turn left/right and stop & 1 \\
\hline & $(35)$ & 3; MI left/right hand and idle state & 3 ; turn left/right and then forward, and stop & 1 \\
\hline & $(43)$ & 2; MI right hand and feet & 2 ; forward and turn right & 1 \\
\hline & (44) & $3 ; \mathrm{MI}$ right/left hand and feet & 3 ; forward and turn left/right & 1 \\
\hline & (46) & $2 ; \mathrm{MI}$ right/left hand or feet & $2 ;$ turn left/right & 1 \\
\hline & $(57)$ & 2; MI right hand and idle state & 7; forward, backward, turn left/right, maintain position and turn on/off the system & 3.5 \\
\hline & (59)a & 3; MI right/left hand and idle state & $27 ; 25$ locations, validate and stop & 9 \\
\hline \multirow{6}{*}{ P300 } & $(27)$ & $1 ;$ selective attention & 9; 7 locations, an "application button" and lock & 9 \\
\hline & (29) & 1 ; selective attention & $18 ; 15$ locations, turn left/right and validate selection & 18 \\
\hline & $(32)$ & 1 ; selective attention & $15 ; 6$ for the BCW (not specified) and 9 for the robotic arm & 15 \\
\hline & (33) & 1 ; selective attention & 4; forward, backward and turn left/right & 4 \\
\hline & $(47)$ & 1 ; selective attention & 7 ; forward, backward, turn left/right $45^{\circ}$ or $90^{\circ}$ and stop & 7 \\
\hline & $(59) \mathrm{b}$ & 1 ; selective attention & $41 ; 37$ locations, validate or delete selection, stop and show extra locations & 41 \\
\hline \multirow{6}{*}{ SSVEP } & $(30)$ & 1 ; selective attention & 4; forward, backward and turn left/right & 4 \\
\hline & $(41)$ & 1 ; selective attention & 5 ; forward, turn left/right and turn on/off the system & 5 \\
\hline & (48) & 1 ; selective attention & 4 ; forward, turn left/right and stop & 4 \\
\hline & $(50)$ & 1 ; selective attention & 4 ; forward, backward and turn left/right & 4 \\
\hline & $(55)$ & 1 ; selective attention & 5 ; forward, backward, turn left/right and stop & 5 \\
\hline & $(58)$ & $1 ;$ selective attention & 5; 4 locations and a "return to the previous window" command & 5 \\
\hline \multirow{5}{*}{$\begin{array}{l}\text { HYBRID- } \\
\text { MENTAL }\end{array}$} & $(31)$ & $\begin{array}{l}\text { 2; MI hand fingers tapping or MI walking and making left/right turns, } \\
\text { and selective attention }\end{array}$ & 10; 9 locations and stop & 5 \\
\hline & (39) & $4 ; \mathrm{MI}$ right/left hand and feet, and selective attention & 4; accelerate, decelerate and turn left/right & 1 \\
\hline & $(49)$ & 1 ; selective attention & 4 ; forward, stop and turn on/off the system & 4 \\
\hline & $(53)$ & $3 ; \mathrm{MI}$ right/left hand movement and selective attention & $\begin{array}{l}8 \text {; forward, turn left/right, accelerate, decelerate, maintain an uniform velocity and turn on/off the } \\
\text { system }\end{array}$ & 2.67 \\
\hline & $(54)$ & 3; MI right/left hand movement and selective attention & 4 ; accelerate, decelerate and turn left/right & 1.33 \\
\hline \multirow{9}{*}{$\begin{array}{l}\text { MUSCLE- } \\
\text { ASSISTED }\end{array}$} & (36) & 4; MI right/left hand and feet, and cheek movement & 4 ; forward, turn left/right and stop & 1 \\
\hline & (37) & 2 ; left/righ hand movements & $2 ;$ turn left/right & 1 \\
\hline & $(38)$ & 3 ; attention, idle state and eye-blinking & $15 ; 13$ directions, forward and stop & 5 \\
\hline & (40) & 4 ; selective attention and 2-4 eye-blinkings & $8 ; 4$ locations, forward, backward and turn left/right & 2 \\
\hline & $(42)$ & 4 ; raise eyebrows, teeth clench on the left/right side and both & 4 ; forward, backward and turn left/right & 1 \\
\hline & $(45)$ & $4 ; \mathrm{MI}$ right/left hand and teeth clench on the left/right side & 4; forward, turn left/right and stop & 1 \\
\hline & $(51)$ & 2 ; close the eyes and keep them open & 4 ; forward, backward and turn left/right & 2 \\
\hline & $(52)$ & $4 ;$ MI right/left hand, selective attention and eye-blinking & 8 ; forward, backward, turn left/right, stop, accelerate, decelerate and maintain position & 2 \\
\hline & $(56)$ & $\begin{array}{l}3 \text {; blink twice, close the eyes and keep them open in six different } \\
\text { directions }\end{array}$ & 8; forward and backward in three directions, validate and stop & 2.67 \\
\hline
\end{tabular}


Table 3. Feature extraction and classification methods used for the brain-controlled wheelchairs compiled in this review.

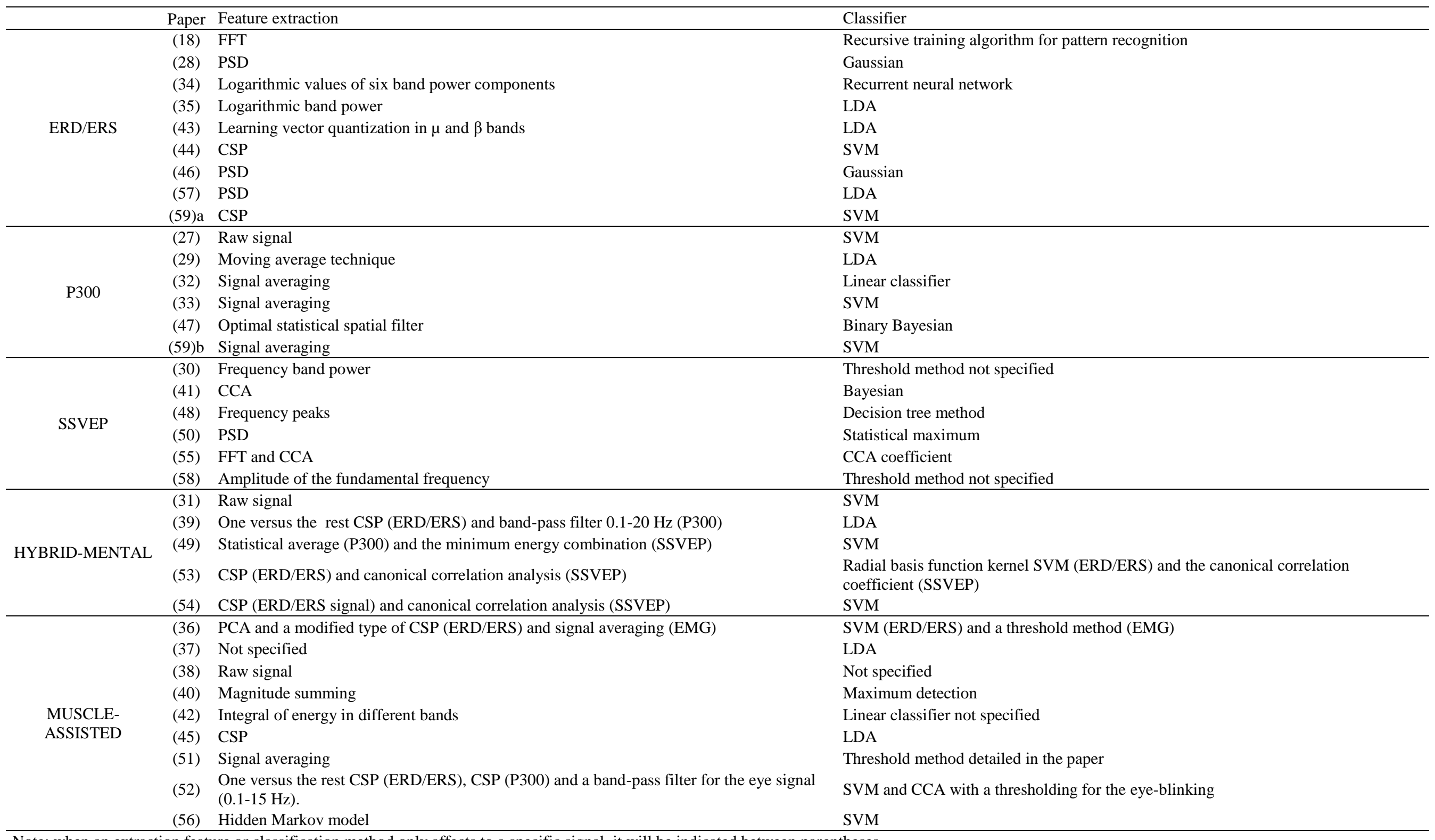

Note: when an extraction feature or classification method only affects to a specific signal, it will be indicated between parentheses. 
Table 4. Used metrics for the evaluation of the BCW.

\begin{tabular}{|c|c|c|}
\hline & Paper & Evaluation \\
\hline \multirow{9}{*}{ ERD/ERS } & $(18)$ & Success rate \\
\hline & (28) & Success rate \\
\hline & (34) & Time required, path length, percentage of hits \\
\hline & $(35)$ & Time required, used commands and collisions \\
\hline & (43) & Percentage of hits \\
\hline & (44) & Percentage of hits (in a standard way and in 4 seconds time windows) \\
\hline & (46) & Path length and time required \\
\hline & $(57)$ & Time required, time optimality rate, used commands, incorrect selections, corrective actions, extra actions and false negatives \\
\hline & $(59) \mathrm{a}$ & Concentration time, incorrect selections, response time to stop, success rate, error distance of the stop area and false activation rate \\
\hline \multirow{6}{*}{ P300 } & $(27)$ & Error rate, selection time and false acceptance rate \\
\hline & (29) & $\begin{array}{l}\text { Task success, path length, time required, path optimality rate, time optimality rate, errors, collisions, used commands, errors caused by a misunderstanding of the interface, } \\
\text { obstacle clearance, number of missions, workload, learnability and confidence }\end{array}$ \\
\hline & $(32)$ & Selection time \\
\hline & (33) & Time required \\
\hline & $(47)$ & Task success, path length, time, path length optimality ratio, time optimality ratio, collisions and success rate \\
\hline & $(59) \mathrm{b}$ & Concentration time, incorrect selections, response time to stop, success rate, error distance of the stop area and false activation rate \\
\hline \multirow{6}{*}{ SSVEP } & $(30)$ & Success rate, best time required and used commands \\
\hline & (41) & Time required \\
\hline & $(48)$ & Qualitative evaluation \\
\hline & $(50)$ & ITR, positive predictive value (PPV) and usability measures \\
\hline & $(55)$ & Unrecognized rate, path length, time required \\
\hline & (58) & Mission: get to reach 4 destinations \\
\hline \multirow{5}{*}{$\begin{array}{l}\text { HYBRID- } \\
\text { MENTAL }\end{array}$} & (31) & Selection time and false positives \\
\hline & (39) & Path length, path optimality rate, time required, classification accuracy, wrong speed control time and collisions \\
\hline & (49) & Missions: to send a "go" command and keep the chair in place, both tasks for 30 seconds \\
\hline & $(53)$ & Time required, useful and useless commands of switch control, selection time and collisions \\
\hline & (54) & Time required and frequency of use of the auxiliary button (to manually avoid collisions) \\
\hline \multirow{9}{*}{$\begin{array}{l}\text { MUSCLE- } \\
\text { ASSISTED }\end{array}$} & (36) & Percentage of hits \\
\hline & (37) & Qualitative evaluation \\
\hline & $(38)$ & Time required \\
\hline & $(40)$ & Success rate, time required and transfer rate (commands per minute) \\
\hline & $(42)$ & Task success, path length, time, used commands, collisions and obstacle clearance (minimum and average distance to the obstacles) \\
\hline & (45) & Time optimality rate \\
\hline & $(51)$ & Success rate and error rate (specified in false positives and false negatives) \\
\hline & $(52)$ & Task success, path length, time required, path length optimality ratio and time optimality ratio \\
\hline & $(56)$ & Task success, path length, time required, path length optimality ratio and time optimality ratio, collisions, mean velocity, workload, learnability, confidence and difficulty \\
\hline
\end{tabular}


signal $(28,46,57)$. Other papers used methods such as learning vector quantization in mu and beta bands (43), the logarithmic value in the bands of interest $(34,35)$, the common spatial patterns (CSP) $(44,59)$ or the fast Fourier transform (FFT) (18). Referring to the classification method, the most used was the followed by support vector machines (SVM) $(44,59)$, Gaussian classifier $(28,46)$, artificial neural networks (34) and a recursive training algorithm for pattern recognition (18).

1.1.1 Evaluation. ERD/ERS systems usually have low-level navigation or shared control, so that the evaluation of a $\mathrm{BCW}$ in real environments is a complex issue, since in most tests the users are asked to go from point $A$ to point $B$, but they are not given the specific commands to reach the destination (figure 3). Therefore, it is difficult to know for sure which commands have been selected or rejected at will (true positives and true negatives, respectively). The metrics most commonly used were success rate, path length, time required, path length optimality ratio, time optimality ratio, number of used commands, and number of collisions, as well as other less common metrics, such as obstacle clearance and command selection time. It is worth mentioning two metrics used by Li and Liang (44), and Varona-Moya (57), which do not take into account the number of hits (or errors) regardless of the runtime, but depend on the user's ability to select each of the commands in a given time, thereby inferring the presence of false negatives if no command is selected. In the proposal of Li and Liang (44), they used a success rate in 4 seconds time window, in which they assumed the intention of generating a command, counting as a mistake not selecting any command. Whereas in the paper of Varona-Moya (57) it was used a metric called missed opportunities, in which false negatives were collected; i.e. cases where the user did not select the optimal command to help them to efficiently complete the course. By other side, in contrast to other BCWs, the proposal of Zhang et al. (59) had a high-level control, so their metrics were a bit different from the rest, may be measured more clearly variables as the success rate or useful in this control as the time to make a selection.

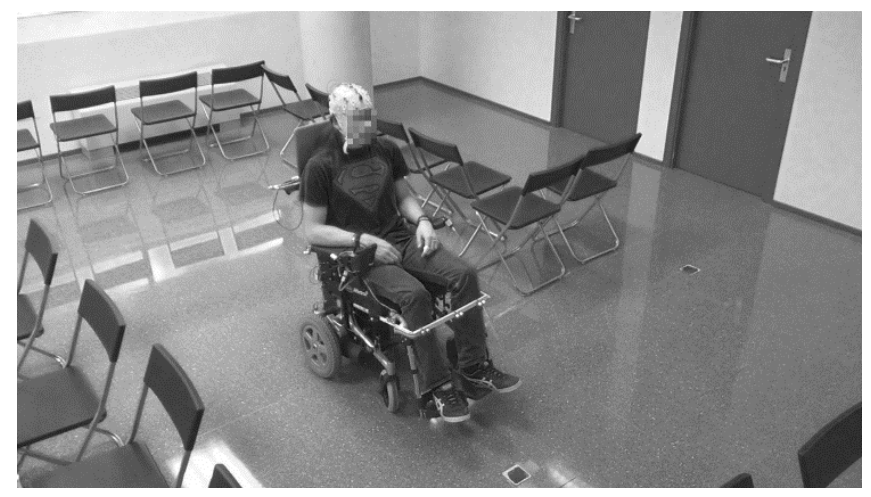

Figure 3. Participant of Varona-Moya et al. (57) during the execution of the path.

\section{$1.2 \mathrm{P} 300 \mathrm{BCWs}$}

P300 is a positive deflection in the voltage of the EEG signal, generally registered from the parietal lobe of the cortex, with a delay of about 300 milliseconds after the presentation of an uncommon target stimulus using an oddball paradigm (2). This paradigm allows the use of a matrix of numerous stimuli, which are selected by visual fixation to execute the command with which they are associated. The main advantages of these systems are: i) they do not require extensive training for management, only a small calibration to adjust the system settings for each user system (7); ii) they tend to have high success rates and iii) high number of available commands, due to the large number of stimuli that these systems allow $(61,62)$. However, a P300 system usually has a low ITR (7) and some studies have highlighted that performance may be reduced in the long term, as the P300 wave amplitude produced by the rare stimulus decreases due to habituation effects (63).

1.2.1 Navigation. Only one P300 BCW used a lowlevel navigation system (33), while three of them used high-level $(31,32,59)$ and two used shared control $(29,47)$. On the one hand, a highlevel system allows the selection of the destination to which the $\mathrm{BCW}$ will go autonomously, so the $\mathrm{P} 300$ is a great candidate to serve as a communication signal, due to its high success rate, and the possibility of offering many destinations in a GUI simultaneously. On the other hand, the only BCW with a low-level navigation system, which was fairly similar to the low-level navigation systems with ERD/ERS signal, based its management on four navigation commands (forward, backward, turn left and turn right), selected through four stimuli in a GUI. The disadvantage of these systems is that they did not allow continuous control of the mobility. By other side, while the shared control proposal of Lopes et al. (47) was quite similar to a low-level navigation system (figure 4), the wheelchair showed by Iturrate et al. (29) had one of the most innovative interfaces, which conducted a mapping of the environment, in which each point was represented in the GUI by a stimulus that could be selected by the user as destination, thus guiding the $\mathrm{BCW}$ to it autonomously. With this system the user gains the flexibility of a low-level navigation in close displacement, with the comfort of a high-level system. 


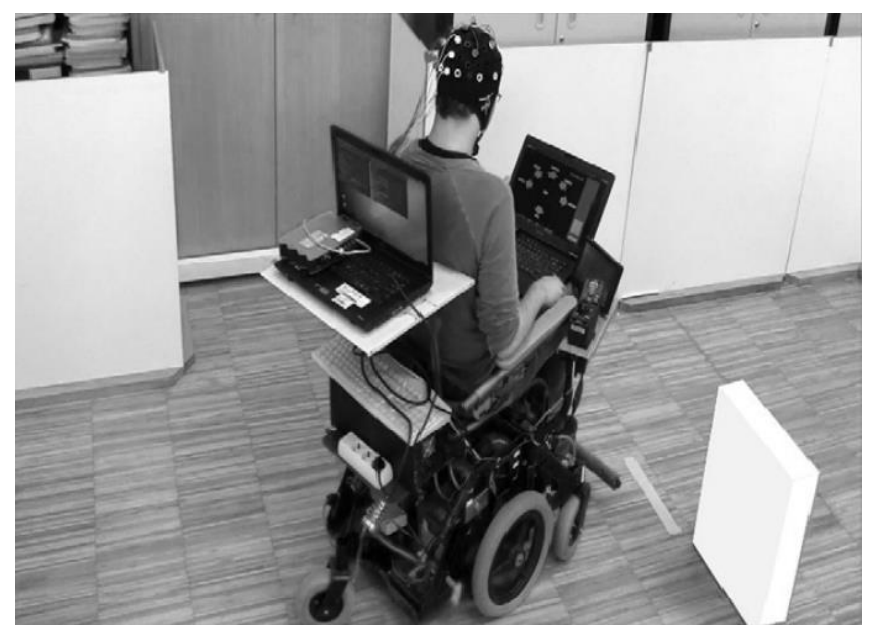

Figure 4. Participant of Lopes et al. (47) in test scenario.

1.2.2 Participants. The average number of participants was $4.83(\sigma=3.71)$. Two papers had at least one disabled user: a patient affected by Guillain-Barre Syndrome (32), and one with cerebral palsy who was severely motorimpaired (47). This latest contribution had the highest number of total users for testing $(\mathrm{N}=$ 11) in P300 BCWs.

1.2.3 Task and interface. P300 interfaces proved very homogeneous, as all BCWs had a GUI to offer their stimuli. The average was 15.5 stimuli $(\sigma=$ 13.07). The only task for the user consists of selective attention to certain stimulus that represents the desired command to be selected and executed by the system. That is, although the number of stimuli may be high, the required task is the same. The average number of commands presented was $15.67(\sigma=13.44)$ so, because the only task used in all studies for selection was the selective attention to the stimulus, the obtained CTR is equal to the number of stimuli (i.e. CTR $=15.67 ; \sigma=13.44$ ).

1.2.4 Feature extraction and classification methods. For P300 signal, the extraction methods were more heterogeneous than the previous ERD/ERS signal. Three proposals used the signal averaging technique $(32,33,59)$, and the others used the moving average (29) and a statistical spatial filter (47). On the other hand, only one BCW used raw signal (27). Referring to the classification methods, the SVM was used by half of the P300's proposals $(27,33,59)$ while the other systems used LDA (29), a binary Bayesian classifier (47) and a not specified linear classifier (32).

1.2.5 Evaluation. Unlike what happened in the BCWs with ERD/ERS signal, in P300 systems the most prevalent navigation was high-level. However, the metrics used in the various articles were very similar, regardless of the navigation system. The most used metrics were: command selection time, hit rate and time required. The paper of Iturrate et al. (29) has the largest number of metrics, which has been referenced many times. This paper used the metrics proposed in Montesano et al. (64) to evaluate the performance of an autonomous system, and had a total of 22 metrics evaluating various factors such as overall performance, command selection, GUI, navigation system, and cognitive variables of the user. Also, in this article, they used two routes: one in which they tested the performance of the $\mathrm{BCW}$ on curved paths, and another for straight and long paths.

\subsection{SSVEP BCWS}

SSVEP signal are cerebral activity modulations produced in the visual cortex by blinking stimulus visualization, at a frequency higher than $4 \mathrm{~Hz}$ (11), eliciting a larger intensity in $5-20 \mathrm{~Hz}$ interval, where most SSVEP BCIs work (62). The main advantages of these systems are: i) high ITR (11); ii) they require short training (65); and iii) they allow an adequate number of commands with good accuracy rates (66). In contrast, some disadvantages are: i) it can provoke fatigue after long-term use; ii) it requires some control of the eye muscles (1); and iii) it can cause epileptic seizures $(1,67)$. The SSVEP signal has been the last kind of signal to be used in a $\mathrm{BCW}$, since it started to be tested with users in 2009 (30).

1.3.1 Navigation. There are three papers about BCWs with low-level navigation $(41,48,50)$, one with high-level navigation (58), and two with shared control $(30,55)$. Although the SSVEP BCWs are based on the selection of a visual stimulus, such as the P300-BCW, we found a clear predominance of prototypical low-level navigation and shared-control systems with four or five commands. This fact could be related to a smaller number of allowed targets in the case of a SSVEP interface, compared to a P300 system. An SSVEP-based BCI can detect how much time users keep their attention on certain stimuli, allowing to maintain the movement as long as users desire; i.e., continuous control (e.g. $(41,50,55))$. Otherwise, the high-level navigation $\mathrm{BCW}$ was similar to the high-level navigation of $\mathrm{P} 300-\mathrm{BCW}$, but with a considerable smaller amount of commands.

1.3.2 Participants. The participant average in SSVEP BCW papers was $5.33(\sigma=4.68)$. The study of $\mathrm{Ng}$ (58) was excluded to calculate the mean, because its number of participants was not representative of the rest $(\mathrm{N}=37)$. By other side, the paper of Diez et al. (50) should be highlighted because it was the only one to include at least one user with MND (a paraplegic patient, with severe paralysis of upper limbs, due to a lesion at the fifth cervical vertebra), and a considerable number of healthy users, with a total of 13 participants.

1.3.3 Task and interface. The number of stimuli is limited with this type of signal, being the average $4.14(\sigma=0.38)$. Most SSVEP-BCWs possessed a low-level navigation system, so the number of commands was relatively low, with an average of $4.67(\sigma=0.52)$, and was generally reduced to five: forward, backward, stop, turn right, and turn left. As was the case in P300 
$\mathrm{BCW}$, there was only one task to be performed by the users, therefore the average value of CTR was equal to the number of commands (CTR $=$ 4.67, $\sigma=0.52$ ).

Most systems used stimuli in the mentioned range of 5 to $20 \mathrm{~Hz}$, with the exception of the study of Diez et al. (50), in which they proved that it is possible to achieve an adequate control of a BCW using high frequencies $(37-40 \mathrm{~Hz})$. The proposal of this study comes from the knowledge that highfrequency flickering stimuli produce less eye strain than low and medium frequencies (68), which is one of the main drawbacks of an SSVEP BCI.

1.3.4 Feature extraction and classification method. The most used feature extraction method was the study of the amplitude in the target frequency $(30,48,58)$, followed by the canonical correlation analysis (CCA) $(41,55)$, the FFT (55) and the PSD (50). As can be seen, the BCW of Duan et al. (55) was the only one using two extraction methods: the CCA and the FFT. On the other hand, the classification methods used were highly heterogeneous, such as the threshold method [31, 58], a statistical classifier (50), a Bayesian classifier (41), the largest CCA coefficient in the case of using this extraction method (55) or a tree decision method used in Müller et al. (48).

1.3.5 Evaluation. In the publications for SSVEP $\mathrm{BCWs}$, the performance evaluation section was the least developed in most of papers, as it was the more heterogeneous area, because they did not observe any metric that was used consistently. However, it is worth distinguishing the use of their own usability questionnaire in Diez et al. (50).

\subsection{Hybrid-mental BCWs}

In this category we will include those BCWs which had a hybrid management; i.e., those which used more than one type of EEG signal (ERD/ERS, P300 and SSVEP) for their control, excluding those that used real movements to control the system. Five papers are included in this category: two ERD/ERS-P300 systems (31,39), two ERD/ERS-SSVEP systems $(53,54)$ and one P300-SSVEP system (49). So it seems to be a trend of using hybrid systems that mix exogenous and endogenous signals, while the use of two exogenous signals is uncommon. The lack of this combination of exogenous systems could be explained because they do not allow the execution of tasks in parallel, since both tasks are selective attention, and the user can only attend to only one stimulus simultaneously. The only BCW that used two exogenous tasks was proposed by Li et al. (49), with the idea of combining both signals to obtain a more reliable system, considering that the selection of a particular command was verified by two different signals.

1.4.1 Navigation. We compiled four BCWs with a low-level navigation system $(39,49,53,54)$, and one with high-level system (31). The proposed high-level BCW by Rebsamen et al. (31) presented a hybrid system in which an MI task was used to stop the BCW, while some destinations were selected through a P300 signal. On the other hand, the low-level navigation BCWs controlled the direction through ERD/ERS signal, while the exogenous signal was used to control the speed. Finally, the only $\mathrm{BCW}$ that was controlled by an exogenous signal could not turn; it just remained in a rest state, or moved forward through the combined use of both exogenous signals, i.e. P300 and SSVEP, to confirm the command.

1.4.2 Participants. The average number of participants was $3.6(\sigma=1.34)$, and there were no patients with MND. The studies were performed with experienced and naïve BCI users. We found the number of participants particularly low again; a fact that is possibly related to the use of ERD/ERS signal for most of these systems.

1.4.3 Task and interface. In these hybrid systems one of the signals must necessarily be exogenous. Therefore the task of visual fixation was present in all these proposals, to allow the selection of a command in a given GUI. The average number of commands presented in this interfaces was $6(\sigma=2.83)$, while the average number of tasks to be performed was $2.6(\sigma=$ 1.14). So, the average CTR obtained was 2.8 ( $\sigma$ $=1.71$ ). Li et al. (54) proposed a BCW based on SSVEP to adjust the speed allowed by selective attention to stimulus, and on MI tasks to control the direction simultaneously, leading to the completion of the path in less time compared with non-hybrid systems. The results were similar in virtual environments in the proposal of Cao et al. (53), using a similar BCW but with a higher number of commands.

1.4.4 Feature extraction and classification methods. In the case of hybrid proposals, they often used a different extraction method for each type of signal. The most used for ERD/ERS signal was the CSP method $(39,53,54)$ while in the case of the SSVEP was the CCA $(53,54)$, with the exception of the proposal of Li et al. (49), which used a minimum energy combination for this signal. Referring the P300 signal, it was filtered at $0.1-20 \mathrm{~Hz}$ in Long et al. (39) and statistically averaged in $\mathrm{Li}$ et al. (49). On the other hand, the proposal of Rebsamen et al. (31) chose not to use any feature extraction method, using the raw signal. For the classification methods, the SVM was used for all hybrid proposals, except for the BCW of Long et al (39), which used the LDA for the analysis of ERD/ERS and P300 signal. Furthermore, while the proposal of Li, Jie et al (53) used SVM for ERD/ERS signal, the largest CCA coefficient was employed for SSVEP signal.

1.4.5 Evaluation. The most common metrics in hybrid systems were time required and those that referred to erroneously or correctly selected commands. However, Li et al. (49) showed an evaluation system that does not require the 
evaluation of a specific path from point $A$ to point $\mathrm{B}$, but consisted of simpler objectives to perform. Such tasks may be more appropriate for evaluating less precise BCWs, which are in an intermediate or initial state of development. However, for more advanced stages of development, where it is necessary to evaluate a more complex performance, it would be necessary to use more demanding tasks for evaluation, taking into account factors such as the classification accuracy of the signal (which is indeed considered in (39) as part of the 'wrong speed control time'), the correct functioning of the navigation system, the GUI, or the user. Like Iturrate et al. (29), Cao et al. (53) used two evaluation environments: one more suitable for the evaluation of straight runs and long distance; and one for curved paths.

\subsection{Muscle-assisted BCWs}

In this category we will include those $\mathrm{BCW}$ shose management is assisted by a muscle signal source. We considered it relevant to include these $\mathrm{BCW}$ in a different classification, as the classic definition of a BCI describe it as "a new non-muscular channel for sending messages and commands to the external world" (1), which does not exactly match the systems presented here, as muscle activity has been used in some way. To improve safety and accuracy of the system, some groups chose to use a muscle signal for handling the interface, either recorded by EMG, EOG, or even the EEG. These interfaces could not be handled by patients with a severe degree of MND, who may also suffer from eye paralysis (ophthalmoplegia) (69). However, these systems can be useful for users that still have residual muscular mobility, as the amplitude of the EMG and EOG signals is much higher than in the case of the EEG signal (70), allowing more precise control of the $\mathrm{BCW}$.

Nine BCWs assisted with muscle signal publications were found: seven were based on an ERD/ERS signal (36$38,42,45,51,56)$, one on P300 signal (40) and one was hybrid of ERD/ERS and P300 signals (52). There was no muscledassisted BCW based on an SSVEP signal. Most of these BCWs used an EEG signal to detect the muscle task. However, two studies used a specific channel to measure muscle signal, as in the case of Li et al. (45), and Wang et al. (52), using EMG and EOG, respectively.

1.5.1 Navigation. Six BCWs used a low-level operation $(37,38,45,51,52,56)$, two high-level $(36,42)$ and one shared control, in which the user could select the type of management by blinking, depending on their needs (40). There was a clear trend to use low-level navigation in muscle-assisted systems, a fact that may be related to the use of an ERD/ERS signal in most of these systems.

1.5.2 Participants. The average number of users per study was $3.78(\sigma=2.59)$, with no MND sufferers. No conclusions can be drawn on the characteristics of participants because many of the studies did not describe any of their features.

1.5.3 Task and interface. The average of total task available to the user was $3.33(\sigma=0.87)$, while the average number of mental and muscle tasks was $1.22(\sigma=1.3)$ and $2.11(\sigma=1.05)$, respectively. Furthermore the number of commands was $6.33(\sigma=3.94)$, resulting in a CTR $=1.96(\sigma=1.3)$. The more-used muscle task was related to blinking or closing the eyes, as well as tasks lateralized as hand movements, or clenching of the right or left side. Most ERD/ERS -based BCIs, including those not using muscular tasks, did not use a GUI for their management, since modulation of these signals does not depend on external stimuli. However, in this category of muscle-assisted BCIs, three articles presented a GUI: the proposal of Lin and Yang (38), in which the user switched between the 13 navigation commands by blinking and selected them by performing a state of attention; the BCW of Wei et al. (42), in which an environment mapping was shown and the user selected the desired position through different muscle tasks (figure 5); and the interface proposed by Ming et al. (51), which showed four stimuli corresponding to the four navigation commands that were illuminated serially, and the participants closed their eyes to select the desired command when it was lit up (figure 6).
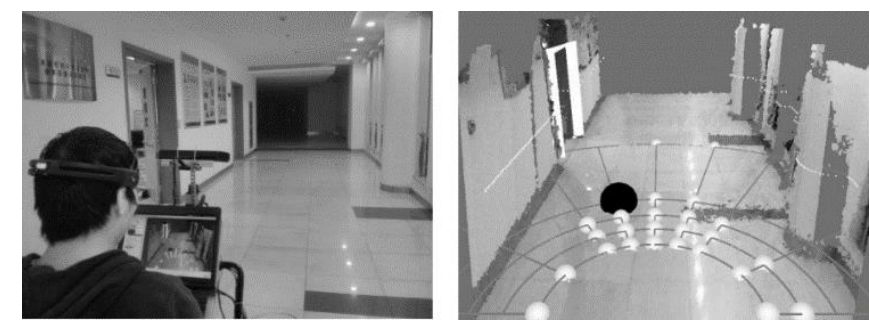

Figure 5. Participant using the BCW of Wei et al. (42).
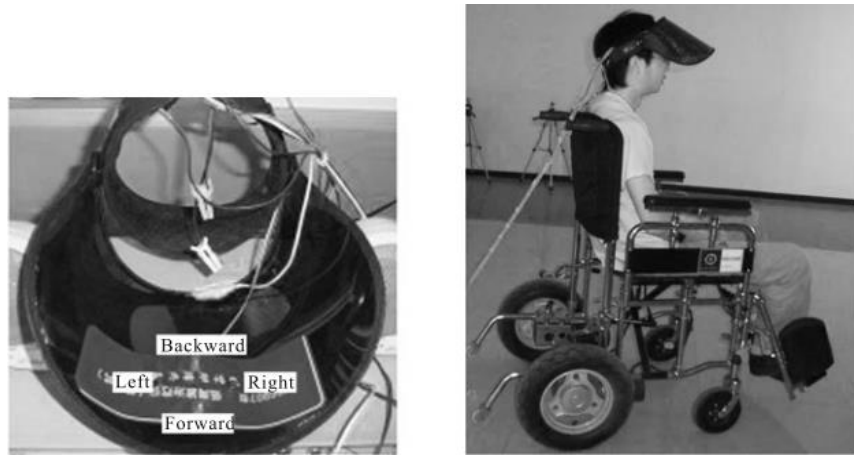

Figure 6. EEG acquisition cap and BCW used by Ming et al. (51).

1.5.4 Feature extraction and classification methods. Although in most proposals of this section the ERD/ERS signal was used besides the muscular movement, the feature extraction methods were diverse: PCA together with CSP to detect MI tasks and actual face movements (36), only CSP to detect the same states (45), the signal averaging to detect blinks or an open/close eyes state $(51,56)$, the integral of energy in different bands to detect different facial movements (42) 
or a hidden Markov model to detect the gaze direction (56). Furthermore, the characteristics of the signal P300 was extracted through CSP in the proposal of Wang et al. (52) while in the BCW of Puanhvuan and Wongsawat (40) a magnitude summing method was presented. However, there was one proposal which used the raw signal, the BCW of (38). On the other hand, about the classification methods, the most used are, again, SVM $(36,52,56)$ and LDA $(37,45)$. Other common method was used, the CCA, however not for the analysis of SSVEP signal but for the eye-blinking detection with a threshold method. There are other proposals with a threshold method (51) or maximum detection found in the proposal of Puanhvuan and Wongsawat (40). The BCW of Choi (36) used EMG in order to generate a stop command through a threshold method where a value was first decided, and if the EMG value exceeds this threshold value, the command execution was stopped.

1.5.5 Evaluation. The most used metric was the success rate, followed by the time required to complete the path. Only two studies included optimized metrics, in terms of time or distance employed to complete the path $(45,52)$. The article with a more complete assessment of muscled-assisted BCWs was published by Wang et al. (52), and was based on some of the metrics used by Iturrate et al. (29).

\section{BCW comparison between categories}

The most common BCWs are those based on ERD/ERS signal, especially if we consider that was the type of signal that included most of the hybrids and muscle-assisted BCWs. Furthermore, we have found a similar amount of BCW papers based purely on P300 and SSVEP signals, both with 18\%. The issue of choosing one signal type or another depends on the need for a signal which allows free control at the expense of a smaller accuracy rate, as in the case of ERD/ERS signals (7). On the other hand, an interface that uses P300 or SSVEP signals requires less training, and achieves higher levels of accuracy (7). The choice of the signal also depends on factors such as: i) the navigation system to be used; ii) the presence or absence of a GUI; iii) the number of commands involved; and iv) the possibility of executing a continuous control of the wheelchair (as in the case of ERD/ERS signals and SSVEP) as opposed to a discrete control (60). Other considerations include the possibility of fatigue produced by SSVEP interfaces, in which an eye strain occurs as the session is extended, or the necessary ability of the user to control their eye muscles (1).

As proposed by Millán et al. (17), as well as in the studies of Li et al. (54) and Cao et al. (53), the use of hybrid systems is recommended, since they offer better results, both in virtual and real environments. The use of commercial EEG devices has only been tested with muscle-assisted systems, where the EEG is used to detect muscle activity. In two of these interfaces, which used the Emotiv EPOC, only muscle movements were used $(37,42)$, while in the case of the NeuroSky ASIC, the authors used eye-blinking and attention state (38). The use of muscle tasks in commercial systems is given by its lower quality signal acquisition, requiring a stronger signal to be detected more easily.

\subsection{Navigation}

Navigation systems based on both ERD/ERS or SSVEP signals were mostly based on low-level control, using the five basic navigation commands: forward, backward, turn left, turn right and stop. P300 BCWs, especially non-hybrids, used mostly high-level control navigation. This may be related to the fact that high-level systems do not allow a step-by-step command selection (so that the user can move freely in the environment), but all possible destinations are predetermined. Therefore, it is necessary that the interface is capable of containing many options that could be chosen to go to a specific place in the environment. Given the existence of numerous commands, the $\mathrm{P} 300$ signal is quickly able to select a command from among others through a similar matrix to the proposed by Farwell and Donchin (2). However, it is noteworthy that the P300 signal, in an oddball paradigm, does not allow the continuous control of the BCW, so in P300 systems, the handling must be necessarily discrete, which may slow the execution of a path; something that does not happen in a system based on an ERD/ERS or SSVEP signal (60).

\subsection{Participants}

The studies with ERD/ERS signals had the lowest number of participants, while for those studies with P300 or SSVEP signals the number of subjects was quite similar. These results can be explained by the difficulty in finding participants with an adequate initial control of these ERD/ERS signals, and the time needed for their training. In addition, even though the end users of this application are people with severe motor disabilities, most studies only tested their systems with healthy participants. Only three studies had disabled participants - two with P300 signal $(32,47)$ and one with SSVEP signal (50) - which are studies based on exogenous signals that do not require training. The use of a large number of participants with previous experience in BCI systems, and subjects that satisfied certain criteria before proceeding to handle the $\mathrm{BCW}$, should be noted.

\subsection{Task and interface}

The number of user tasks for ERD/ERS BCWs is higher compared to systems with an exogenous signal. In contrast, the number of commands, especially for systems based on $\mathrm{P} 300$, is higher in exogenous systems. Therefore, as expected, the BCWs with a higher CTR are those based on P300 ( $\bar{x}=$ 15.67; $\sigma=13.44)$, followed by those based on $\operatorname{SSVEP}(\bar{x}=$ 4.67; $\sigma=0.52)$, hybrids-mental $(\bar{x}=2.8 ; \sigma=1.71)$, muscleassisted $(\bar{x}=2 ; \sigma=1.32)$ and ERD/ERS $(\bar{x}=2 ; \sigma=2.54)$. The lower number of commands allowed in ERD/ERS systems could be explained because each user's task typically execute a particular command - how it is reflected in the CTR - and, how it was mentioned, fewer tasks to classify involve a better performance classification $(25,26)$.

Referring to the user tasks to be performed by participants, in systems that include an exogenous signal, there is only one task - selective attention to the stimulus whereas in those with an ERD/ERS signal the most used task was MI, especially left - or right - hand MI. The use of a 
GUI was required in SSVEP or P300-based systems, but it was not necessary in most of the interfaces using ERD/ERS signal. Thanks to the absence of GUI in most ERD/ERS systems, users do not need to focus on a screen while they drive the $\mathrm{BCW}$, allowing them to keep their attention on the environment, and to be alert to possible unexpected events. Most hybrid systems combined exogenous and endogenous signals, as it was not common to use more than one type of exogenous signal, as was indeed the case for $\mathrm{Li}$ et al. (49). The combination of endogenous and exogenous signals allowed the execution of more than one command simultaneously; e.g. a selective attention visual task and MI $(53,54)$. Regarding the number of visual stimuli presented in a GUI, the P300 interfaces $(\bar{x}=15.5, \sigma=13.07)$ obtained a larger number than those based on an SSVEP signal $(\bar{x}=4.14$, $\sigma=0.38)$.

\subsection{Feature extraction and classification methods}

Firstly, respect to the feature extraction methods, a great variety can be seen, from papers using CSP, PSD or signal averaging, to those which use raw signal. It is difficult to show a clear pattern about what is the most used method, but it is observed a tendency to use the PSD with the ERD/ERS signal, the signal averaging techniques in P300 and the CCA in SSVEP. On the other hand, referring to the classification methods, less variety has been found, being mainly used LDA and SVM thanks to its own properties. First, LDA requires a low computational load which allows a suitable online control of the BCI system though its low performance on the analysis of non-linearly separable data. By other side, SVM offers better generalization properties, and it is insensitive to overtraining and to the curse-of-dimensionality; however, it provides a lower speed execution (71). This methods were followed by Gaussian and Bayesian classifiers, artificial neural network, the coefficient obtained in the CCA or a specific thresholding method. Furthermore, it should be noted that some of these papers use a thresholding method or a linear classifier without specifying how they are calculated.

\subsection{Evaluation}

The evaluation criteria used in the different studies were very heterogeneous, from the simplest with just a couple of metrics, to the most elaborate exceeding ten. In reference to the signal, those BCWs based on ERD/ERS or SSVEP signal systems gave greater importance to the evaluation of accuracy in the selection of commands, and the time required to complete the path. However, in the P300 BCWs, half of the studies used the selection time as their metric, defined as the time it took the user to select the desired command. This metric could hardly be controlled on ERD/ERS-based BCWs because it is difficult to know when the user starts the command selection during the free handling of the wheelchair.

\section{Conclusions}

Thirty-four papers were found which show the progress made to control a wheelchair through the registration of a user's brain activity. The trend to use an EEG signal in BCI applications is shown to be more pronounced in a $\mathrm{BCW}$, being the only one used, possibly due to the difficulty of adapting the necessary instrumentation of other techniques in a vehicle that allows user displacement. However, it is necessary to improve some features to be able to ameliorate the daily life of patients with MND.

As mentioned, the use of each type of EEG signal carries many advantages and disadvantages that must be considered. In addition, new proposals for BCWs should offer more flexible systems that are easier to learn and use, which enable their fluid, natural, and safe control. Indeed, current trends include the use of hybrid BCIs, which have been shown to allow: i) a faster management of the $\mathrm{BCW}$ through performing tasks in parallel, allowing the execution of two commands simultaneously $(53,54)$; or ii) improved accuracy by combining two signals (49). However, some objections identified in most papers should be noted. Despite the advantages of using navigation systems that assist the control of the wheelchair with shared control, only $25.71 \%$ of all papers collected had this aid. On the other hand, there was almost no study with users affected by some type of MND, despite these being the end users for this kind of application. Therefore, for future proposals, it is desirable to test the management of the BCWs by users affected by a MND, to face the new challenges that this might involve. It may be recommended to increase the total number of participants in the studies, because the interest should not be simply to show that a proposal is capable of running a $\mathrm{BCW}$ with an adequate performance, but to find systems that any user can manage, not just those users with excellent skills.

Referring to the evaluation systems, as proposed by Bi et al. (20), it could be of interest to use similar metrics among different papers, so that the proposals can be compared more accurately. Moreover, only a few articles presented the use of metrics related to the user's experience during handling. These metrics should be collected through questionnaires, regarding the usability of workload or fatigue, using a standardized test whenever possible. Concerning the usability issue, one of the major objections that users often report after a BCI experience is the discomfort caused by the use of the EEG cap, and the electrode conductive gel (72). These objections may be aggravated in the case of patients suffering from MND, which increases the complication in the EEG device montage. Therefore, we recommend studying the possibility of acquiring a suitable control for a $\mathrm{BCW}$ using commercial EEG systems which exist currently. In addition, the economic gap between the commercial devices and those normally used in the laboratory can be the difference between the elitist use of such devices, or one that everybody could afford.

Despite these points for improvement, studies have evolved positively in various aspects. Some examples are the following: flexibles navigation systems which assists the user during their displacement; studies with more than 10 participants, as well as the presence of patients with some MND; innovative proposals appear with the possibility of simultaneous speed and direction management of the BCW serial interfaces that allow a high CTR with ERD/ERS, or even by a purely auditory or visual signal interface; and there is the development of metrics to an extensive evaluation of $\mathrm{BCW}$ and adapted to its specific characteristics. Indeed, all these contributions lead us to firmly believe that BCWs may be used safely and effectively in the near future for a patient with MND, at least in controlled environments. However, before $\mathrm{BCWs}$ could be used in real outdoor environments, it 
will be necessary to better guarantee the adequate users' control and the system safety.

To conclude, the goal of a useful BCW is definitely an ambitious project that is being developed through the work of several research groups, which are closer to benefit and improve the quality of life of people with MND despite the many challenges to overcome.

\section{Acknowledgments}

This research was partially supported, by the Spanish Ministry of Economy and Competitiveness through the projects INCADI (TEC2011-26395) and LICOM (DPI201567064-R), and by the European Regional Development Fund (ERDF).

\section{References}

1. Wolpaw J, Birbaumer N, McFarland DJ, Pfurtscheller G, Vaughan TM. Brain Computer Interfaces for communication and control. Clin Neurophysiol. 2002;113:767-91.

2. Farwell LA, Donchin E. Talking off the top of your head: Toward a mental prosthesis utilizing eventrelated brain potentials. Electroencephalogr Clin Neurophysiol. 1988;70(6):510-23.

3. Hochberg LR, Bacher D, Jarosiewicz B, Masse NY, Simeral JD, Vogel J, et al. Reach and grasp by people with tetraplegia using a neurally controlled robotic arm. Nature [Internet]. 2012;485(7398):372-5. Available from: http://www.nature.com/nature/journal/v485/n7398/f ull/nature 11076.html

4. Leeb R, Tonin L, Rohm M, Desideri L, Carlson T, Millán JDR. Towards Independence: A BCI Telepresence Robot for People With Severe Motor Disabilities. Vol. PP, Proceedings of the IEEE. 2015. p. 1-14.

5. Corralejo R, Hornero R, Álvarez D. A domotic control system using Brain-Computer Interface (BCI). 2011. 345-352 p. (Lecture Notes in Computer Science (including subseries Lecture Notes in Artificial Intelligence and Lecture Notes in Bioinformatics); vol. 6691 LNCS).

6. Daly JJ, Wolpaw JR. Brain-computer interfaces in neurological rehabilitation. Lancet Neurol. 2008;7(11):1032-43.

7. Nicolas-Alonso LF, Gomez-Gil J. Brain Computer Interfaces, a Review. Sensors [Internet]. 2012;12(12):1211-79. Available from: http://www.mdpi.com/1424-8220/12/2/1211/

8. Kleber B, Birbaumer N. Direct brain communication: neuroelectric and metabolic approaches at Tübingen. Cogn Process [Internet]. 2005;6(1):65-74. Available from: http://dx.doi.org/10.1007/s10339-004-0045-8
9. Hinterberger T, Schmidt S, Neumann N, Mellinger J, Blankertz B, Curio G, et al. Brain-Computer Communication and Slow Cortical Potentials. IEEE Trans Biomed Eng. 2004;51(6):1011-8.

10. Friedrich EVC, Scherer R, Neuper C. The effect of distinct mental strategies on classification performance for brain-computer interfaces. Int $\mathrm{J}$ Psychophysiol [Internet]. Elsevier B.V.; 2012;84(1):86-94. Available from: http://dx.doi.org/10.1016/j.ijpsycho.2012.01.014

11. Vialatte F-B, Maurice M, Dauwels J, Cichocki A. Steady-state visually evoked potentials: focus on essential paradigms and future perspectives. Prog Neurobiol [Internet]. 2010 Apr [cited 2015 Nov 18];90(4):418-38. Available from: http://www.sciencedirect.com/science/article/pii/S03 01008209001853

12. Thakor N. Biopotentials and Electrophysiology Measurement. In CRC Press; 1999. Available from: http://dx.doi.org/10.1201/9780415876179.ch74

13. Taylor GS, Schmidt C. Empirical Evaluation of the Emotiv EPOC BCI Headset for the Detection of Mental Actions. Proc Hum Factors Ergon Soc Annu Meet [Internet]. 2012 Sep 1;56 (1 ):193-7. Available from:

http://pro.sagepub.com/content/56/1/193.abstract

14. Vourvopoulos A, Liarokapis F. Evaluation of commercial brain-computer interfaces in real and virtual world environment: A pilot study. Comput Electr Eng [Internet]. Elsevier Ltd; 2014;40(2):714$29 . \quad$ Available from: http://www.sciencedirect.com/science/article/pii/S00 45790613002577

15. Ron-Angevin R, Díaz-Estrella A. Brain-computer interface: Changes in performance using virtual reality techniques. Neurosci Lett. 2009;449(2):1237.

16. van de Laar B, Gurkok H, Bos DP-O, Poel M, Nijholt A. Experiencing BCI Control in a Popular Computer Game. Comput Intell AI Games, IEEE Trans. 2013;5(2):176-84.

17. Millán JDR, Rupp R, Müller-Putz GR, Murray-Smith $\mathrm{R}$, Giugliemma C, et al. Combining brain-computer interfaces and assistive technologies: state-of-the-art and challenges. Front Neurosci [Internet]. 2010;4(SEP):1-15. Available from: http://journal.frontiersin.org/article/10.3389/fnins.20 10.00161/abstract

18. Tanaka K, Matsunaga K, Wang HO. Electroencephalogram-based control of an electric wheelchair. IEEE Trans Robot. 2005;21(4):762-6.

19. Millán JDR, Renkens F, Mouriño J, Gerstner W. Noninvasive brain-actuated control of a mobile robot by human EEG. IEEE Trans Biomed Eng [Internet]. 2004;51(6):1026-33. Available from: http://www.ncbi.nlm.nih.gov/pubmed/15188874 
20. Bi L, Fan X-A, Liu Y. EEG-Based Brain-Controlled Mobile Robots: A Survey. IEEE Trans HumanMachine Syst [Internet]. 2013;43(2):161-76. Available from: http://ieeexplore.ieee.org/lpdocs/epic03/wrapper.ht $\mathrm{m}$ ?arnumber $=6461528$

21. Neuper C, Pfurtscheller G. Neurofeedback Training for BCI Control. In: Graimann B, Pfurtscheller G, Allison B, editors. Brain-Computer Interfaces [Internet]. Springer Berlin Heidelberg; 2010. p. 6578. Available from: http://dx.doi.org/10.1007/978-3642-02091-9_4

22. Vanhooydonck D, Demeester E, Nuttin M, Van Brussel H. Shared control for intelligent wheelchairs: an implicit estimation of the user intention. Int Work Adv Serv Robot [Internet]. 2003;176-82. Available from:

http://citeseerx.ist.psu.edu/viewdoc/download?doi=1 0.1.1.83.6885\&amp;rep=rep1\&amp;type=pdf

23. Demeester E, Nuttin M, Vanhooydonck D, Brussel H Van. A model-based, probabilistic framework for plan recognition in shared wheelchair control: experiments and evaluation. Proc 2003 IEEE/RSJ Int Conf Intell Robot Syst (IROS 2003) (Cat No03CH37453). 2003;2(October):1456-61.

24. Amiri S, Fazel-Rezai R, Asadpour V. A review of hybrid brain-computer interface systems. Adv Human-Computer Interact. 2013;2013.

25. Kronegg J, Chanel G, Voloshynovskiy S, Pun T. Eegbased synchronized brain-computer interfaces: A model for optimizing the number of mental tasks. IEEE Trans Neural Syst Rehabil Eng. 2007;15(1):50-8.

26. Obermaier B, Neuper C, Guger C, Member A, Pfurtscheller G. Information Transfer Rate in a FiveClasses Brain-Computer Interface. 2001;9(3):283-8.

27. Rebsamen B, Burdet E, Guan C, Teo CL, Zeng Q, Ang M, et al. Controlling a wheelchair using a BCI with low information transfer rate. 2007 IEEE 10th Int Conf Rehabil Robot ICORR'07 [Internet]. 2007;1003-8. Available from: http://dx.doi.org/10.1109/ICORR.2007.4428546

28. Millán JDR, Galán F, Vanhooydonck D, Lew E, Philips J, Nuttin M. Asynchronous non-invasive brain-actuated control of an intelligent wheelchair. Proc 31st Annu Int Conf IEEE Eng Med Biol Soc Eng Futur Biomed EMBC 2009 [Internet]. 2009;2009:3361-4. Available from: http://www.ncbi.nlm.nih.gov/pubmed/19963794

29. Iturrate I, Antelis JM, Kubler A, Minguez J. A Noninvasive Brain-Actuated Wheelchair Based on a P300 Neurophysiological Protocol and Automated Navigation. IEEE Trans Robot. 2009;25(3):614-27.

30. Mandel C, Lüth T, Laue T, Röfer T, Gräser A, KriegBrückner B, et al. Navigating a smart wheelchair with a brain-computer interface interpreting steady-state visual evoked potentials. 2009 IEEE/RSJ Int Conf Intell Robot Syst IROS. 2009;1118-25.

31. Rebsamen B, Guan C, Zhang H, Wang C, Teo C, Jr. MHA, et al. A brain controlled wheelchair to navigate in familiar environments. IEEE Trans Neural Syst Rehabil Eng. 2010;18(6):590-8.

32. Alqasemi R, Dubey R. A 9-DoF WheelchairMounted Robotic Arm System: Design, Control, Brain-Computer Interfacing, and Testing. In: Hall E, editor. Advances in Robot Manipulators. InTech; 2010 .

33. Shin B-G, Kim T, Jo S. Non-invasive brain signal interface for a wheelchair navigation. In: Control Automation and Systems (ICCAS), 2010 International Conference on. 2010. p. 2257-60.

34. Hema CR, Paulraj MP, Yaacob S, Adom AH, Nagarajan R. Software Tools and Algorithms for Biological Systems. 2011;696:565-72. Available from: http://link.springer.com/10.1007/978-1-44197046-6

35. Tsui CSL, Gan JQ, Hu H. A Self-Paced Motor Imagery Based Brain-Computer Interface for Robotic Wheelchair Control. Clin EEG Neurosci. 2011;42(4):225-9.

36. Choi K. Control of a vehicle with EEG signals in realtime and system evaluation. Eur J Appl Physiol [Internet]. 2012;112(2):755-66. Available from: http://www.ncbi.nlm.nih.gov/pubmed/21667185

37. Carrino F, Dumoulin J, Mugellini E, Khaled OA, Ingold R. A self-paced BCI system to control an electric wheelchair: Evaluation of a commercial, lowcost EEG device. In: Biosignals and Biorobotics Conference (BRC), 2012 ISSNIP. 2012. p. 1-6.

38. Lin J-S, Yang W-C. Wireless brain-computer interface for electric wheelchairs with EEG and eyeblinking signals. Int J Innov Comput Inf Control. 2012;8(9):6011-24.

39. Long J, Li Y, Wang H, Yu T, Pan J, Li F. A Hybrid Brain Computer Interface to Control the Direction and Speed of a Simulated or Real Wheelchair. Neural Syst Rehabil Eng IEEE Trans. 2012 Sep;20(5):720 9 .

40. Puanhvuan D, Wongsawat Y. Semi-automatic P300based brain-controlled wheelchair. In: Complex Medical Engineering (CME), 2012 ICME International Conference on. 2012. p. 455-60.

41. $\mathrm{Xu} \mathrm{Z,} \mathrm{Li} \mathrm{J,} \mathrm{Gu} \mathrm{R,} \mathrm{Xia} \mathrm{B.} \mathrm{Steady-State} \mathrm{Visually}$ Evoked Potential (SSVEP)-Based Brain-Computer Interface (BCI): A Low-Delayed Asynchronous Wheelchair Control System. Neural Inf Process Iconip 2012, Pt I [Internet]. 2012;7663:305-14. Available from: <Go to ISI >://WOS:000345086000037

42. Wei Z, Chen W, Wang J, Wang H, Li K. Semantic 
mapping for safe and comfortable navigation of a brain-controlled wheelchair. Lect Notes Comput Sci (including Subser Lect Notes Artif Intell Lect Notes Bioinformatics). 2013;8102 LNAI(PART 1):307-17.

43. Carra M, Balbinot A. Evaluation of sensorimotor rhythms to control a wheelchair. In: Biosignals and Biorobotics Conference (BRC), 2013 ISSNIP. 2013. p. 1-4.

44. Li J, Liang J. Design of assistive wheelchair system directly steered by human thoughts. Int J Neural Syst. 2013;23(3):1-12.

45. Li Z, Lei S, Su C-Y, Li G. Hybrid brain/muscleactuated control of an intelligent wheelchair. In: Robotics and Biomimetics (ROBIO), 2013 IEEE International Conference on. 2013. p. 19-25.

46. Carlson T, Millán JDR. Brain-controlled wheelchairs: A robotic architecture. IEEE Robot Autom Mag. 2013;20(1):65-73.

47. Lopes AC, Pires G, Nunes U. Assisted navigation for a brain-actuated intelligent wheelchair. Rob Auton Syst [Internet]. Elsevier B.V.; 2013;61(3):245-58. Available from: http://dx.doi.org/10.1016/j.robot.2012.11.002

48. Müller SMT, Bastos TF, Filho MS. Proposal of a SSVEP-BCI to Command a Robotic Wheelchair. J Control Autom Electr Syst [Internet]. 2013;24(1):97105. Available from: http://dx.doi.org/10.1007/s40313-013-0002-9

49. Li Y, Pan J, Wang F, Yu Z. A hybrid BCI system combining P300 and SSVEP and its application to wheelchair control. IEEE Trans Biomed Eng. 2013;60(11):3156-66.

50. Diez PF, Müller SMT, Mut VA, Laciar E, Avila E, Bastos-Filho TF, Sarcinelli-Filho MM. Commanding a robotic wheelchair with a high-frequency steadystate visual evoked potential based brain-computer interface. Med Eng Phys [Internet]. Institute of Physics and Engineering in Medicine; 2013;35(8):1155-64. Available from: http://dx.doi.org/10.1016/j.medengphy.2012.12.005

51. Ming D, Fu L, Chen L, Tang J, Qi H, Zhao X, et al. Electric wheelchair control system using braincomputer interface based on alpha-wave blocking. Trans Tianjin Univ [Internet]. 2014;20(5):358-63. Available from: http://dx.doi.org/10.1007/s12209014-2235-5

52. Wang H, Li Y, Long J, Yu T, Gu Z. An asynchronous wheelchair control by hybrid EEG-EOG braincomputer interface. Cogn Neurodyn. 2014;399-409.

53. Cao L, Li J, Ji H, Jiang C. A hybrid brain computer interface system based on the neurophysiological protocol and brain-actuated switch for wheelchair control. J Neurosci Methods [Internet]. Elsevier B.V.; 2014;229:33-43. Available from: http://dx.doi.org/10.1016/j.jneumeth.2014.03.011
54. Li J, Ji H, Cao L, Zang D, Gu R, Xia B, et al. Evaluation and Application of a Hybrid Brain Computer Interface for Real Wheelchair Parallel Control With Multi-Degree of Freedom. Int J Neural Syst [Internet]. 2014;24(04):1450014. Available from:

http://www.worldscientific.com/doi/abs/10.1142/S0 129065714500142

55. Duan J, Li Z, Yang C, Xu P. Shared control of a brain-actuated intelligent wheelchair. In: Intelligent Control and Automation (WCICA), 2014 11th World Congress on. 2014. p. 341-6.

56. Aziz F, Arof H, Mokhtar N, Mubin M. HMM based automated wheelchair navigation using EOG traces in EEG. J Neural Eng [Internet]. IOP Publishing; 2014;11(5):056018. Available from: http://www.scopus.com/inward/record.url?eid=2s2.0-84907908970\&partnerID=tZOtx3y1

57. Varona-Moya S, Velasco-Álvarez F, Sancha-Ros S, Fernández-Rodríguez Á, Blanca MJ, Ron-Angevin R. Wheelchair Navigation with an Audio-cued, TwoClass Motor Imagery-based Brain - Computer Interface System . 7th International IEEE/EMBS Conference on Neural Engineering (NER). 2015;224.

58. Ng DW-K, Soh Y-W, Goh S-Y. Development of an Autonomous BCI Wheelchair. 2014 IEEE Symp Comput Intell Brain Comput Interfaces [Internet]. 2014;1-4. Available from: http://www.scopus.com/inward/record.url?eid=2s2.0-84923042171\&partnerID=tZOtx3y1

59. Zhang R, Li Y, Yan Y, Zhang H, Wu S, Yu T, et al. Control of a Wheelchair in an Indoor Environment Based on a Brain-Computer Interface and Automated Navigation. Neural Syst Rehabil Eng IEEE Trans [Internet]. 2015;PP(99):1. Available from: http://ieeexplore.ieee.org/lpdocs/epic03/wrapper.ht $\mathrm{m}$ ?arnumber $=7115145$

60. Fazel-Rezai R, Allison BZ, Guger C, Sellers EW, Kleih SC, Kübler A. P300 brain computer interface: current challenges and emerging trends. Front Neuroeng [Internet]. 2012;5(July):14. Available from:

http://www.pubmedcentral.nih.gov/articlerender.fcgi ?artid $=3398470 \&$ tool $=$ pmcentrez\&rendertype $=$ abstr act

61. Townsend G, LaPallo BK, Boulay CB, Krusienski DJ, Frye GE, Hauser CK, et al. A novel P300-based brain-computer interface stimulus presentation paradigm: Moving beyond rows and columns. Clin Neurophysiol [Internet]. Elsevier; 2010;121(7):1109-20. Available from: http://dx.doi.org/10.1016/j.clinph.2010.01.030

62. Wang Y, Wang R, Gao X, Hong B, Gao S. A practical VEP-based brain-computer interface. IEEE Trans Neural Syst Rehabil Eng [Internet]. 2006;14(2):2349. Available 
http://www.ncbi.nlm.nih.gov/pubmed/16792302

63. Ravden D, Polich J. On P300 measurement stability: habituation, intra-trial block variation, and ultradian rhythms. Biol Psychol [Internet]. 1999 Oct [cited 2016 Apr 29];51(1):59-76. Available from: http://www.sciencedirect.com/science/article/pii/S03 01051199000150

64. Montesano L, Minguez J, Alcubierre JM, Montano L. Towards the adaptation of a robotic wheelchair for cognitive disabled children. Intell Robot Syst 2006 IEEE/RSJ Int Conf. 2006;710-6.

65. Gao X, Xu D, Cheng M, Gao S. A BCI-based environmental controller for the motion-disabled. IEEE Trans Neural Syst Rehabil Eng. 2003;11(2):137-40.

66. Bin G, Gao X, Yan Z, Hong B, Gao S. An online multi-channel SSVEP-based brain-computer interface using a canonical correlation analysis method. J Neural Eng [Internet]. 2009;6(4):46002. Available from: http://stacks.iop.org/1741$2552 / 6 / \mathrm{i}=4 / \mathrm{a}=046002$

67. Fisher RS, Harding G, Erba G, Barkley GL, Wilkins A. Photic- and pattern-induced seizures: A review for the epilepsy foundation of america working group. Epilepsia. 2005;46(9):1426-41.

68. Diez PF, Mut V a, Avila Perona EM, Laciar Leber E. Asynchronous BCI control using high-frequency SSVEP. J Neuroeng Rehabil [Internet]. BioMed Central Ltd; 2011;8(1):39. Available from: http://www.jneuroengrehab.com/content/8/1/39

69. Takeda S, Yamada M, Kawasaki K, Oyanagi K, Ikuta F, Arai M, et al. Motor neuron disease with multisystem involvement presenting as tetraparesis, ophthalmoplegia and sensori-autonomic dysfunction. Acta Neuropathol [Internet]. 1994;88(3):193-200. Available from: http://dx.doi.org/10.1007/BF00293393

70. McMenamin BW, Shackman AJ, Greischar LL, Davidson RJ. Electromyogenic Artifacts and Electroencephalographic Inferences Revisited. Neuroimage [Internet]. 2011 Jan 1;54(1):4-9. Available from: http://www.ncbi.nlm.nih.gov/pmc/articles/PMC296 $2711 /$

71. Lotte F, Congedo M, Lécuyer A, Lamarche F, Arnaldi B. A review of classification algorithms for EEG-based brain-computer interfaces. Journal of Neural Engineering. 2007;4(2)

72. Holz EM, Höhne J, Staiger-Sälzer P, Tangermann M, Kübler A. Brain-computer interface controlled gaming: Evaluation of usability by severely motor restricted end-users. Artif Intell Med [Internet]. Elsevier; 2016 Apr 30;59(2):111-20. Available from: http://dx.doi.org/10.1016/j.artmed.2013.08.001 